\title{
Multiscale air quality with the NMMB/BSC Chemical Transport Model
}

Jorba, O.' , Pérez, C. ${ }^{2}$, Janjic, Z. ${ }^{3}$, Baldasano, J.M. ${ }^{1,4}$, Dabdub, D.5, Badia, A. ${ }^{1}$, Spada, M. ${ }^{1}$,Haustein, K. ${ }^{1}$

1 Earth Sciences Department, Barcelona Supercomputing Center, Barcelona, Spain 2Earth Institute at Columbia University, NASA GISS, New York, USA

${ }^{3} \mathrm{NOAA} / \mathrm{NWS}$ /National Centers for Environmental Prediction, Camp Springs, MD, USA 4Environmental Modeling Laboratory, Technical University of Catalonia, Barcelona, Spain ${ }^{5}$ Department of Mechanical and Aerospace Engineering, University of California Irvine, USA 


\section{Motivation: BSC air quality modeling activities}

- CALIOPE daily forecast and verification

$\checkmark$ Daily experimental forecasts for meteorology and air quality (12 $\mathrm{km}$ for Europe and $4 \mathrm{~km}$ for the Iberian Peninsula) (http://www.bsc.es/caliope).
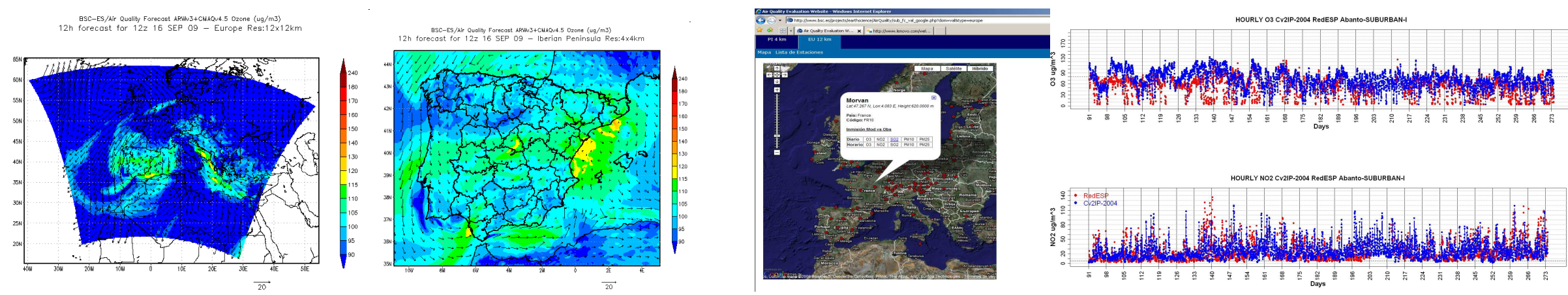

- BSC-DREAM8b daily forecast and verification

North Africa/Mediterranean - $1 / 3 \times 1 / 3$ degree resolution Asia domain - $1 / 2 \times 1 / 2$ degree resolution
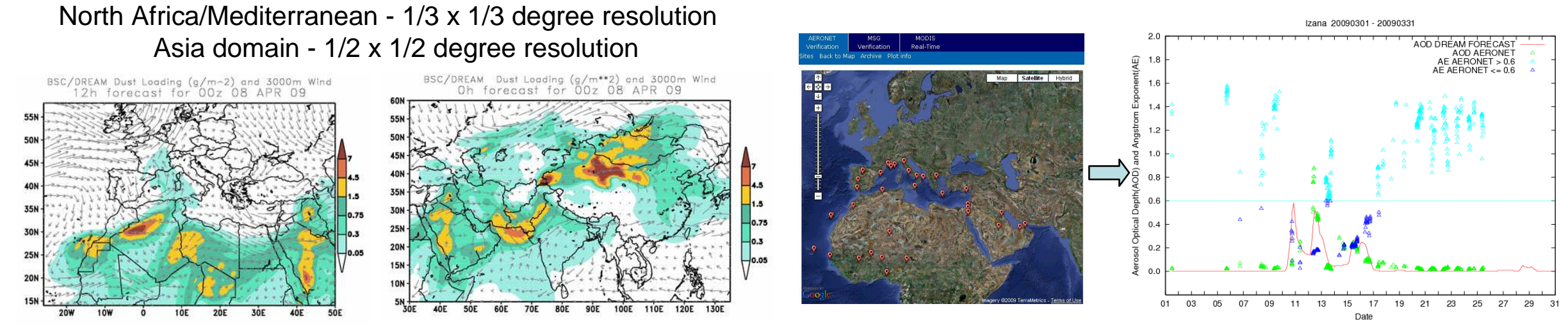

$\rightarrow$ http://www.bsc.es/projects/earthscience/DREAM 


\section{Historical evolution}

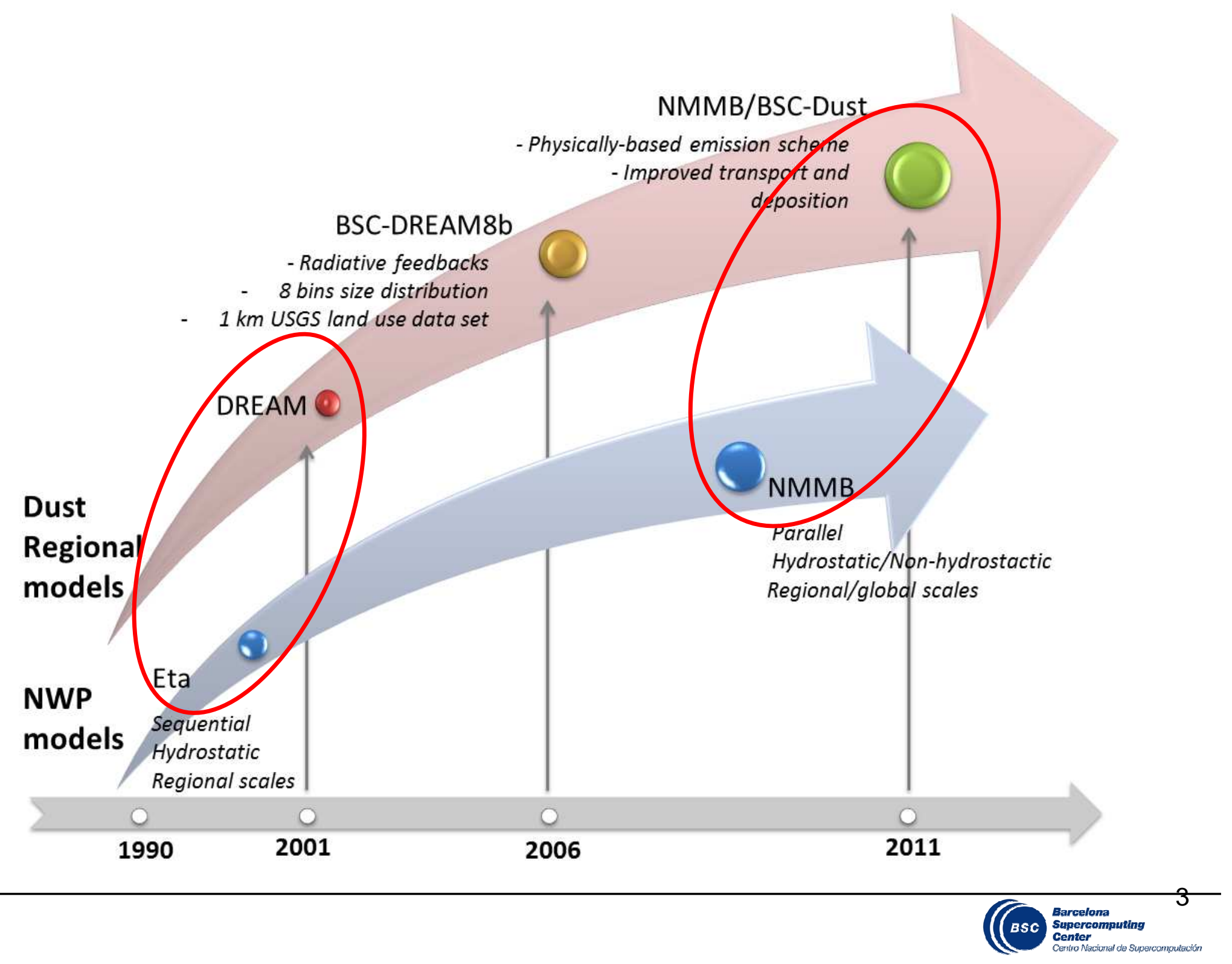




\section{NMMB/BSC-Chemical Transport Model}

\section{Embedding dust and chemistry processes within the meteorological core driver NMMB}

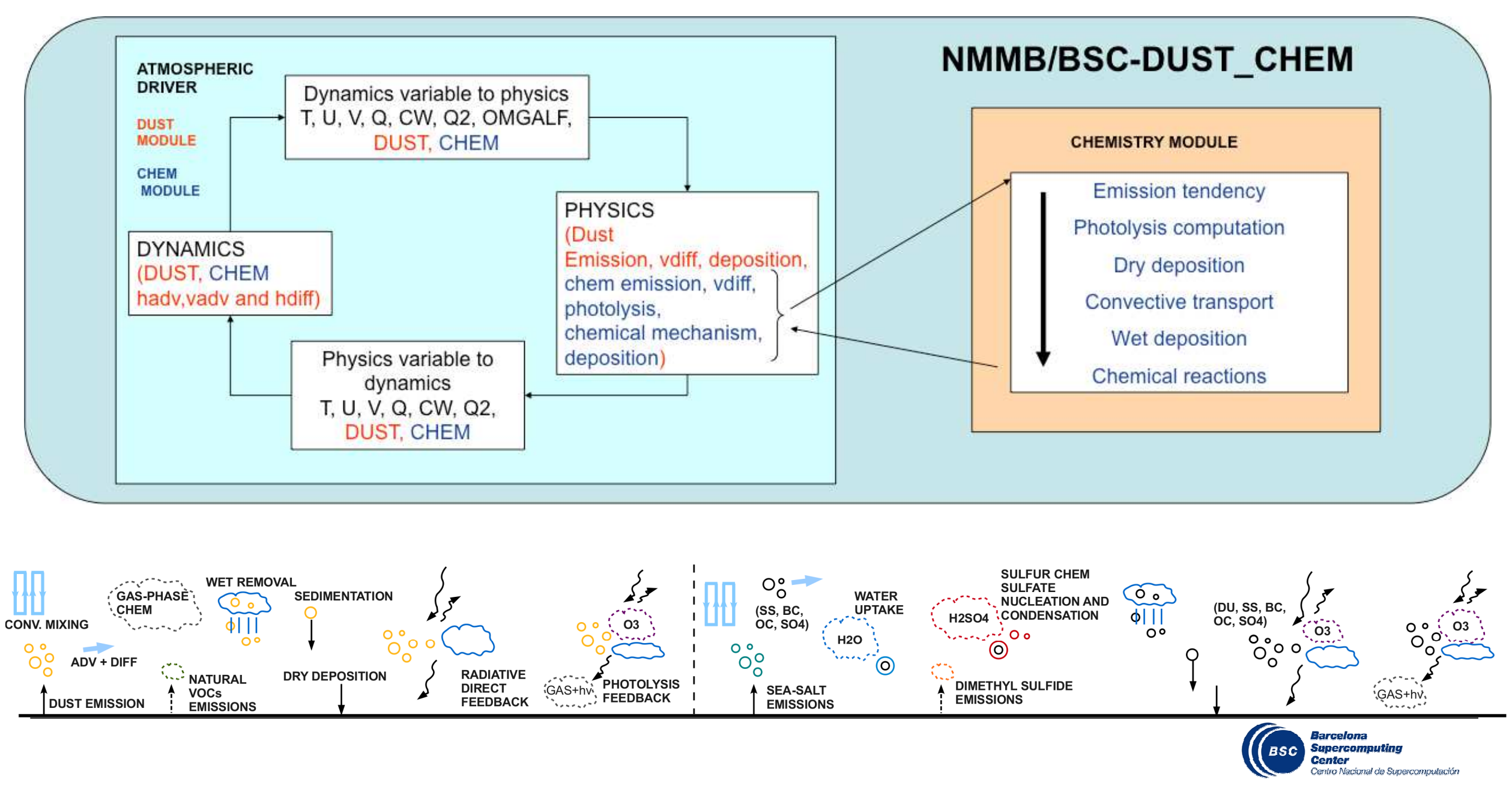


NMMB - Nonhydrostatic Multiscale Model on the B grid - Main characteristics

Under development at NCEP (Janjic, 2005; Janjic, 2007; Janjic, 2009; Janjic et al., 2011)

\section{Unified nonhydrostatic dynamical core (list of features is not exhaustive)}

$\checkmark$ Wide range of spatial and temporal scales (from meso to global)

$\checkmark$ Regional and global domains (just a simple switch)

$\checkmark$ Evolutionary approach,built on NWP experience by relaxing hydrostatic approximation $>$ Favorable features of the hydrostatic formulation preserved

$\checkmark$ The nonhydrostatic option as an add-on nonhydrostatic module

$\checkmark$ No problems with weak stability on mesoscales

$\checkmark$ Conservation of important properties of the continuous system

$\checkmark$ Arakawa B grid (in contrast to the WRF-NMM E grid)

$\checkmark$ Pressure-sigma hybrid

$\checkmark$ Improved tracer advection: Eulerian, positive definite, mass conservative and monotonic

$\checkmark$ NMMB regional became the next-generation NCEP mesoscale model for operational weather forecasting in 2011 


\section{NMMB/BSC-Chemical Transport Model}

- Mineral Dust module - NMMB/BSC-Dust (Pérez et al., 2011; Haustein et al., 2012)

- Evolution of the BSC-DREAM8b model (Nickovic et al., 2001; Pérez et al., 2006)

- Implementation of all common on-line dust modules for global and regional simulations

- Current DREAM dust emission scheme upgraded to a physically based scheme (explicitly accounting for saltation and sandblasting)

- New high resolution database for soil textures and vegetation fraction

- Direct radiative effect implemented

- Gas phase chemistry (Jorba et al, 2012)

- Integrated implementation within NMMB - chemistry solved after NMMB physics

- Consistent advection and diffusion schemes with meteorology

- Feedback interactions aerosols-photolysis allowed

- Processes implemented online: emission, chemistry, dry and wet deposition each timestep. Online biogenic emissions from MEGAN

- Global relevant aerosol module (spada et al., 2012)

- Complementing NMMB/BSC-DUST mineral dust aerosols

- Same numerics like dust implementation

- Inclusion of Sea Salt, BC, OC and sulfate

- Implementation of feedbacks forseen 


\section{NMMB/BSC-CTM: gas-phase chemistry processes (Jorba et al., 2009-2012; Badia and Jorba, 2011)}

\section{Photolysis scheme}

- On-line Fast-J scheme (Wild et al., 2000)

- Coupled with physics of each model layer (e.g., aerosols, clouds). Planned to couple with NMMb/BSCDUST aerosols.

- Considers NMMB grid-scale clouds and NMMB/BSCCHEM O3 or climatology

- 7 bins wave-length (quick version)

$$
J_{i}=\int_{\lambda_{1}}^{\lambda_{2}} F(\lambda) \sigma_{i}(\lambda) \phi_{i}(\lambda) d \lambda \quad \begin{array}{ll} 
& F(\lambda): \text { actinic flux } \\
& \sigma_{i}(\lambda): \text { absorption cross section } \\
& \Phi_{i}(\lambda): \text { quantum yield of phot. react. }
\end{array}
$$

- Tables of $\sigma_{i}(\lambda)$ and $\Phi_{i}(\lambda)$ to be updated from Prather Fast-JX.

\section{Dry deposition}

- Wesely et al. $(1986,1989)$ implemented to compute deposition velocities

- $\quad$ Simple scheme coupled with surface model layer physics (e.g., skin temperature, incoming shortwave radiation, friction velocity, ...)

- Solve dry deposition in chemistry module independently from vertical diffusion. Considering to solve dry depositoin and vertical diffusion at first model level at same time.

$$
\begin{gathered}
d C_{i}\left(z_{r e f}\right) / d t=-V_{g}\left(z_{r e f}\right) \times C_{i}\left(z_{r e f}\right) / \Delta z \\
V_{d}=\left(R_{a}+R_{b}+R_{c}\right)^{-1}
\end{gathered}
$$

\section{Chemical mechanism}

- $\quad$ CBM-IV and CB05 mechanisms implemented (Gery et al., 1989; Yarwood, 2005)

- Coupled with Fast-J photolysis scheme

- Mechanism implemented through KPP kinetic preprocesor (Damian et al., 2002)

- KPP coupling allows a straightforward modification of chemistry kinetics and reactions. Suitable for sensitivity studies.

- Implemented an EBI solver for CB05

- Stratospheric ozone: linear model Cariolle and Teyssèdre (2007) or Monge-Sanz et al. (2011)

\section{Cloud chemistry}

- $\quad$ Cloud chemistry includes: scavenging, mixing, wet deposition and aqueous chemistry

- $\quad$ Scavenging and wet deposition implemented for gridscale and sub-gridscale clouds following Byun and Ching (1999)

- Sub-grid + gridscale:

Scavenging: $\left.\frac{\partial \bar{m}_{i}}{\partial t}\right|_{c l d}=\left.\frac{\partial \bar{m}_{i}}{\partial t}\right|_{\text {subcld }}+\left.\frac{\partial \bar{m}_{i}}{\partial t}\right|_{\text {rescld }}$

- Wet deposition:

$w d e p_{i}=\int_{0}^{\tau_{c l d}} \bar{m}_{i}^{c l d} P_{r} d t$
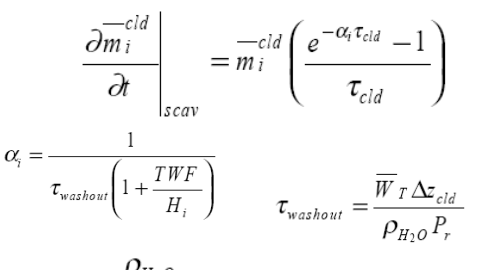

$T W F=\frac{\rho_{H_{2} O}}{\bar{W}_{T} R T}$ 


\section{NMMB/BSC-DUST: dust processes}

\section{(Pérez et al., 2008-2011; Haustein et al., 2009-2012)}

- NMMb/BSC-DUST emission scheme

$\rightarrow$ Soil moisture effects [Fecan et al., 1999] $\quad$ (DREAM + NMMb-DUST)
$u_{\text {set }_{\text {wet }}}=u_{\text {*dry }} \cdot \sqrt{1+1.21 \cdot\left(w-w^{\prime}\right)^{0.68}}$
$\Longrightarrow w^{\prime}=(0.0014 \% \text { clay })^{2}+0.17 \%$ clay
$w^{\prime}=$ max amount of adsorbed water

$\rightarrow$ Drag partition correction [Marticorena and Bergametti, 1995]

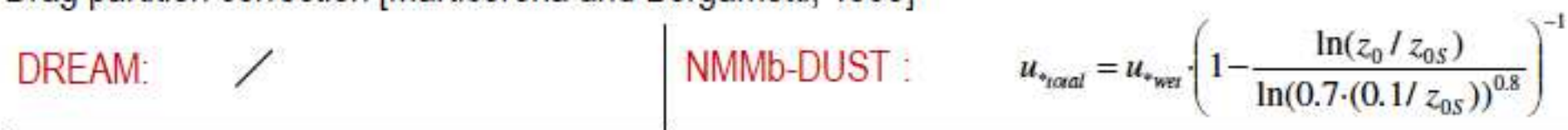

$\rightarrow$ Threshold friction velocity [Bagnold, 1941; Iversen and White, 1982; Marticorena and Bergametti, 1995]

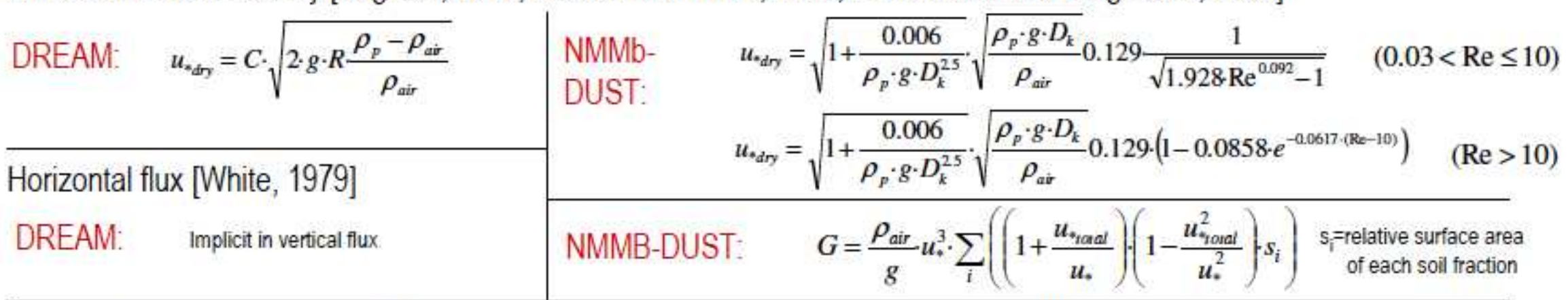

$\rightarrow$ Vertical flux [Shao et al., 1993; Marticorena and Bergametti, 1995; Tegen et al., 2002]

DREAM: $\quad F_{S}=c \cdot \delta_{\text {DREMM }} \cdot u_{*}^{3} \cdot\left(1-\frac{u_{\text {ronal }}^{2}}{u_{*}}\right) \quad$ NMMb-DUST: $\quad F_{s}=c \cdot \alpha \cdot \delta \cdot G \quad \Longrightarrow \quad\left(u_{*} \geq u_{\text {trotal }}\right)$

$\rightarrow$ Viscous sublayer effects near the surface [Janjic, 1994] $\quad$ (DREAM + NMMb-DUST)

$F_{S Z 0}=K_{S}^{\prime} \cdot \frac{C_{L M}-C_{0}}{\Delta z} \quad \Longrightarrow \quad K_{S}^{\prime}=\frac{1}{1+\omega} K_{S} \quad K_{5}=$ diffusion coefficient, $\omega=$ weighting factor 


\section{NMMB/BSC-DUST: dust processes}

\section{(Pérez et al., 2008-2011; Haustein et al., 2009-2012)}

$\rightarrow$ Turbulent deposition [Giorgi, 1986] (DREAM + NMMb-DUST)

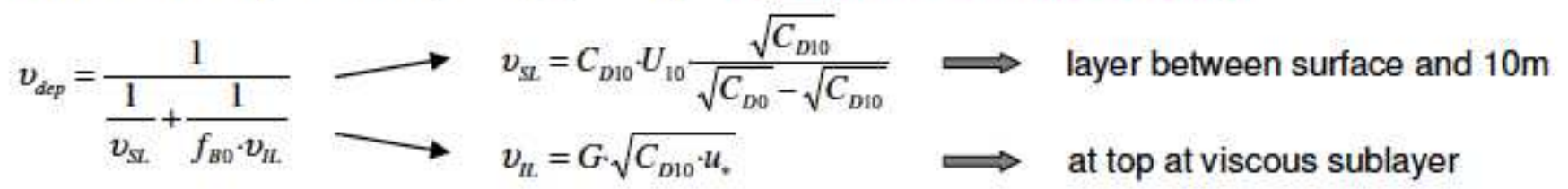

$\rightarrow$ factor $\mathrm{G}$ accounts differently for surface with turbulent regime and surface covered by vegetation

$\rightarrow$ in the latter case accounting for Brownian diffusion, interception, impaction and small vegetation elements

$\rightarrow$ Gravitational settling [Giorgi, 1986]

$$
\text { DREAM: } \quad v_{g}=\frac{2 \cdot g \cdot \rho_{k} \cdot R_{k}^{2}}{9 \cdot v}
$$

$$
\text { NMMb-DUST: } \quad v_{s}=\frac{2 \cdot g \cdot \rho_{k} \cdot R_{k}^{2}}{9 \cdot v} C c
$$

$$
C c=1+\frac{2 \cdot \lambda}{R_{k}} \cdot\left(1.257+0.4 e^{\frac{-0.55 \cdot R_{k}}{\lambda}}\right) \quad \text { Cunningham correction }
$$

$\rightarrow$ Grid scale precipitation [Slinn, 1983; 1984]

DREAM: Simple below cloud wasout ratio for grid-scale precipitation (Zhao microphysics)
$\mathrm{NMMb-DUST:} \mathrm{In-cloud} \mathrm{scavenging} \mathrm{from} \mathrm{grid-scale} \mathrm{clouds}$ and Below-cloud scavenging from grid-scale precipitation (snow and rain) (Ferrier microphysics)

$\rightarrow$ Convective precipitation [Loosmore and Cederwall, 2004]

DREAM: below cloud wasout ratio for convective precipitation (Betts-Miller-Janjic)
NMMb-DUST:

In-cloud and below-cloud scavenging for convective precipitation (Betts-Miller-Janjic) 


\section{NMMB/BSC-CTM: Sea Salt processes (Spada et al., 2012)}

- Sectional approach - 8 bins for coarse and fine SSA

- Assumed dry density of $2160 \mathrm{~kg} / \mathrm{m}^{3}$, solubility factors derived from Zender et al. (2003)

- Hygroscopic growth following Chin et al. (2002)

- Affecting gravitational settling, dry and wet deposition

- Different open-ocean sea-salt emission schemes implemented and tested.

- Monahan et al. (1986) -> bubbles

- Gong (2003) -> bubbles

- Smith et al. (1993) -> bubbles, spume

- Martensson et al. (2003) -> bubbles (SST dependent)

sea-salt emission schemes: number flux parameterization at $\mathrm{U}_{10}=9 \mathrm{~m} / \mathrm{s}$

- Combined M86/SM93 -> bubbles, spume

- Combined MA03/M86/SM93 -> bubbles, șpume, SST dependent

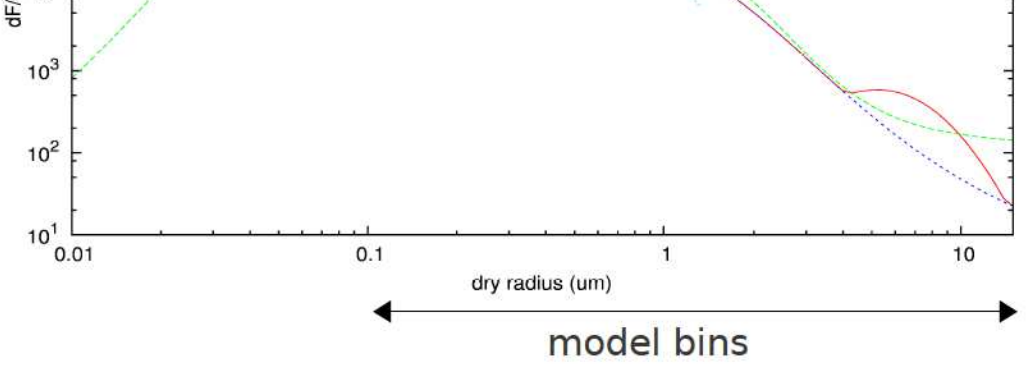

model bins 
- Results and evaluation works

- Dust

- Gas phase chemistry

- Sea salt 


\section{Results: Dust model}

\section{Pa.}

- Global and regional annual simulations evaluated with:

- Aeronet sun-photometer networks

- LIDAR vertical profiles
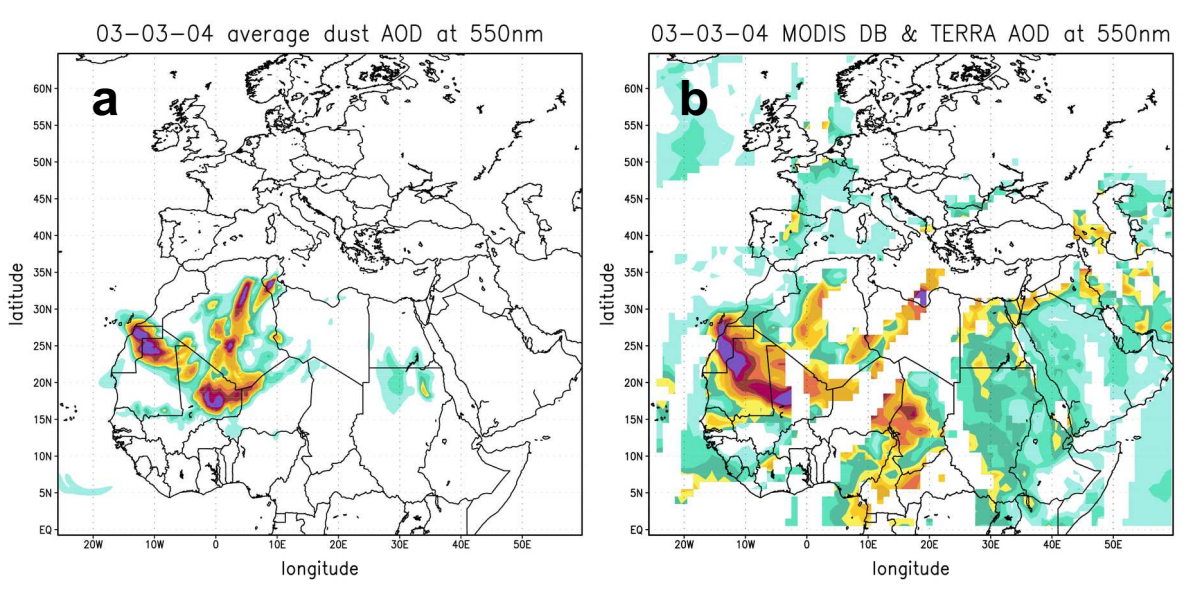

- Several satellite products

- Surface concentrations

- Emission and deposition fluxes
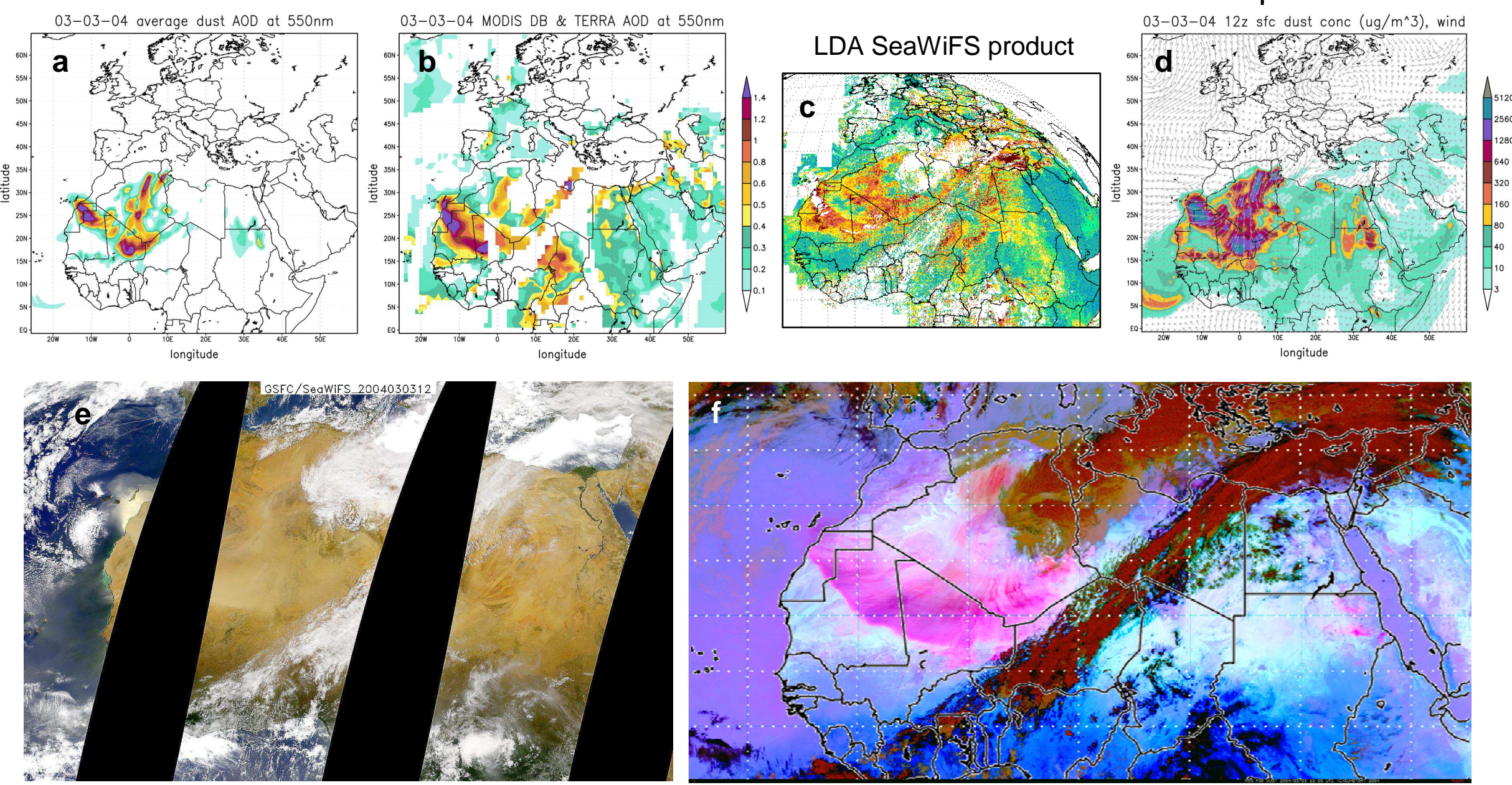

Pérez et al., 2011; Haustein et al., 2012

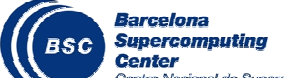




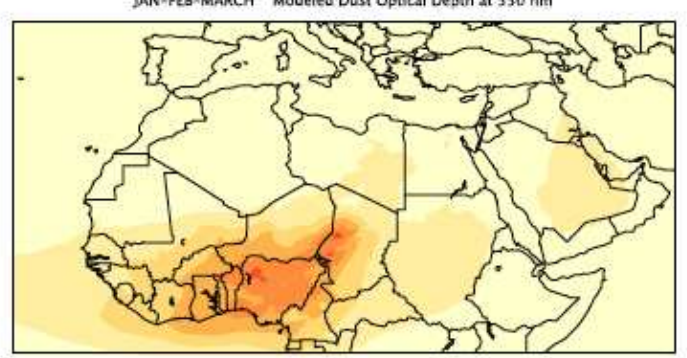

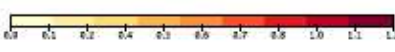
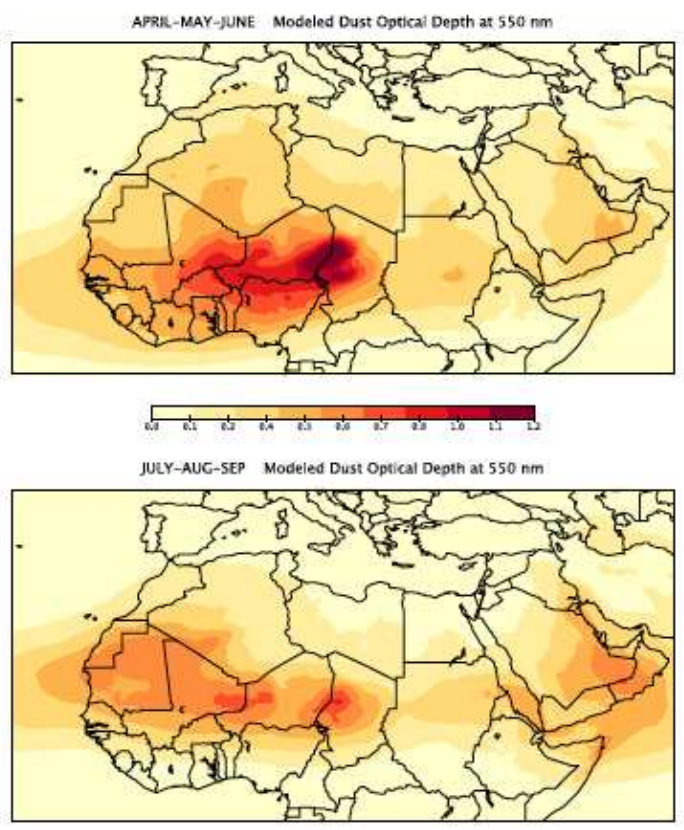

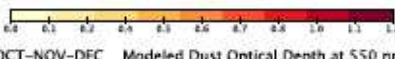

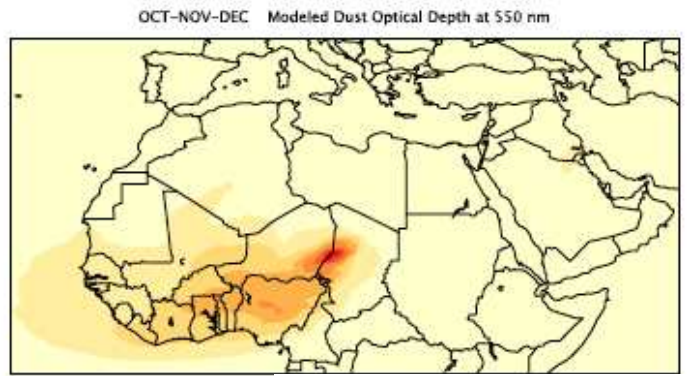

AN-

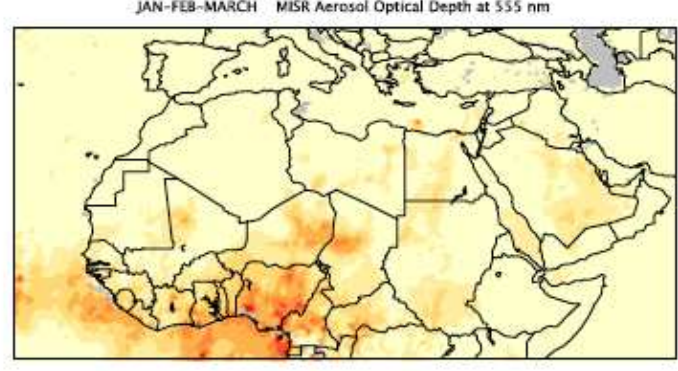

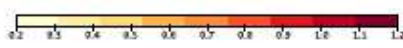

APRLL-MAY-JUNE MSR Aerosol Oogtcal Degth at $555 \mathrm{~nm}$

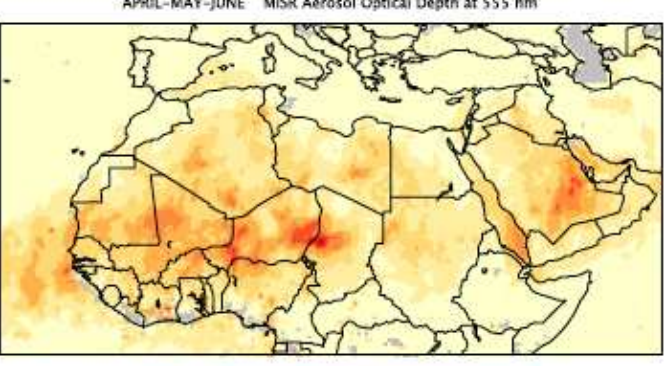

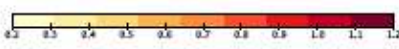

JULY-AUG-SEP MISR Aerosol Optical Depth at $555 \mathrm{~nm}$

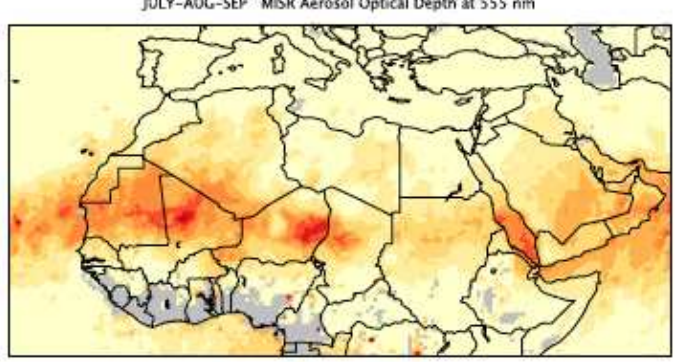

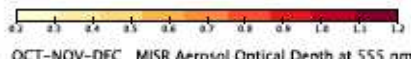

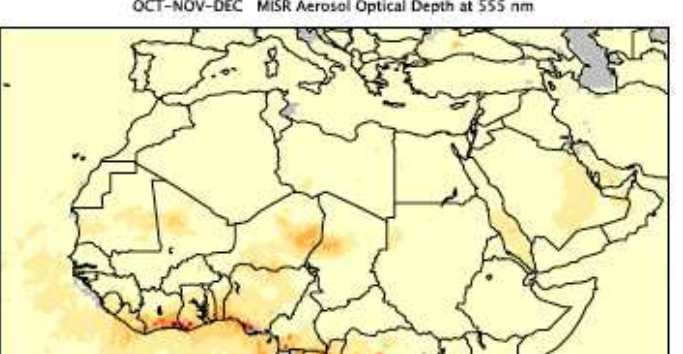

IAN-FEB-MARCH OMI Aerosol Index
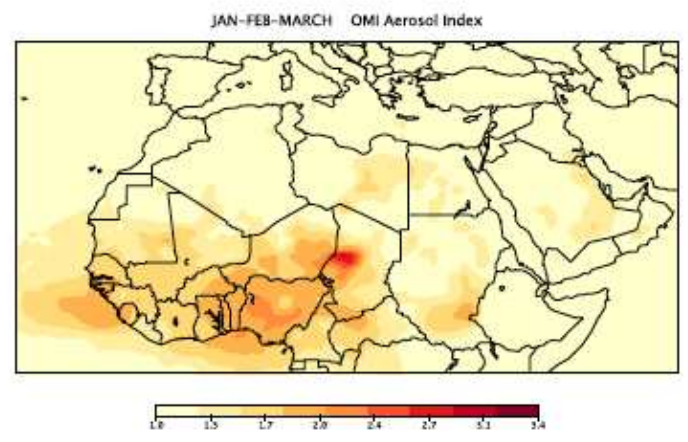

APRLL-MAY-JUNE OM! Aerosol Indere

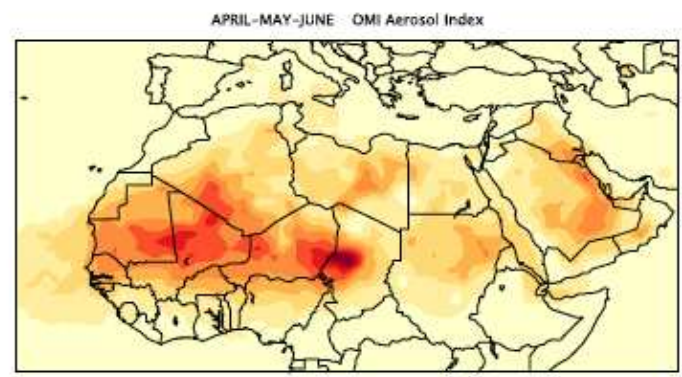

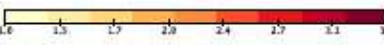

JULY-AUC-SEP OMI Aerosol Index
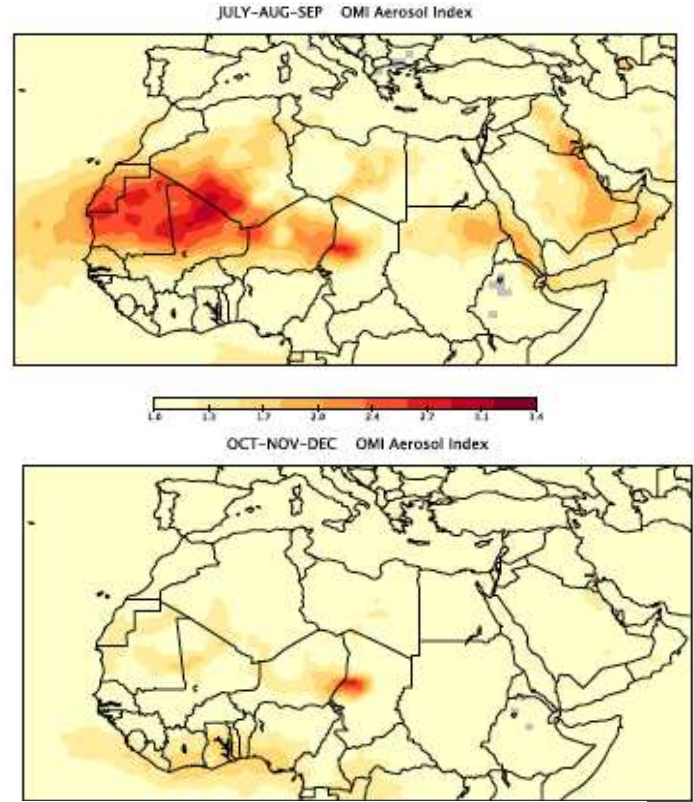
Ilorin

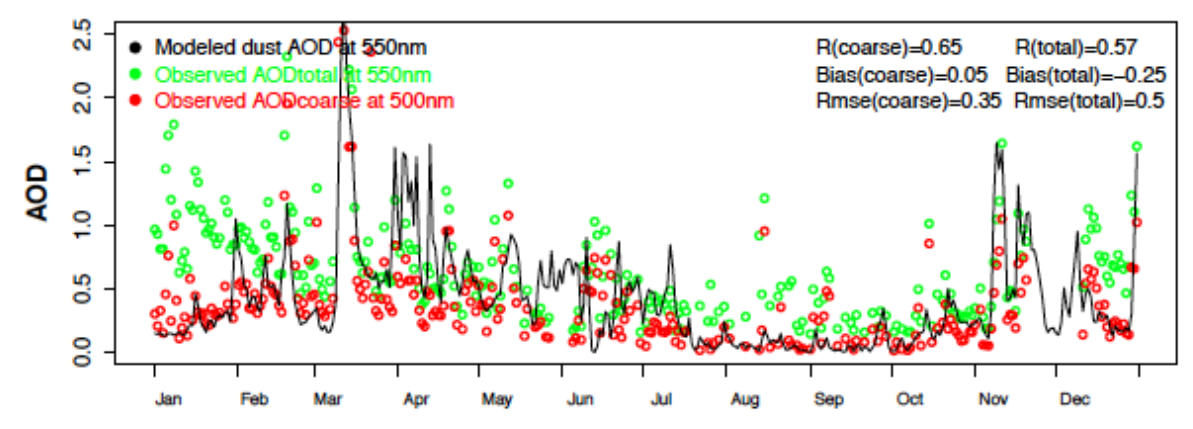

Agoufou

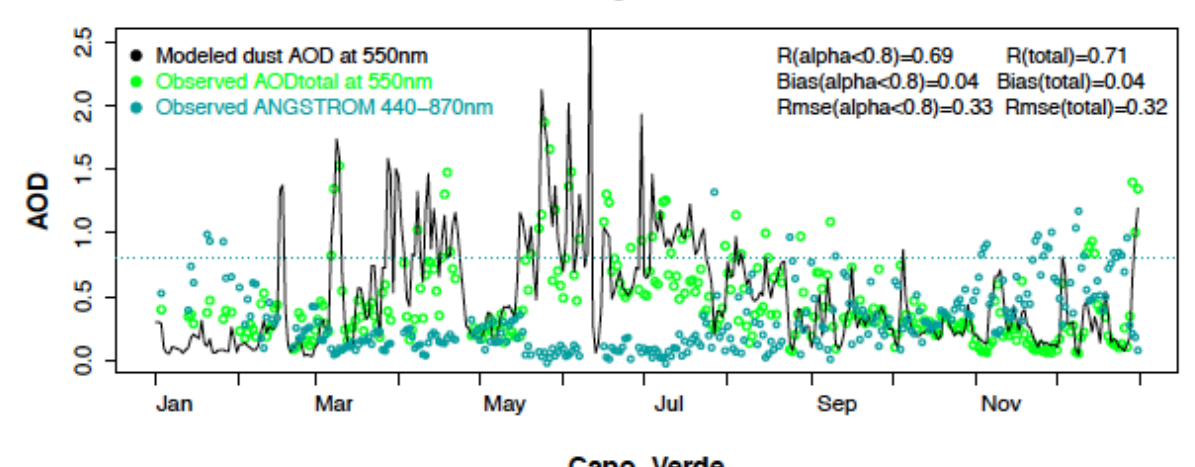

Capo_Verde

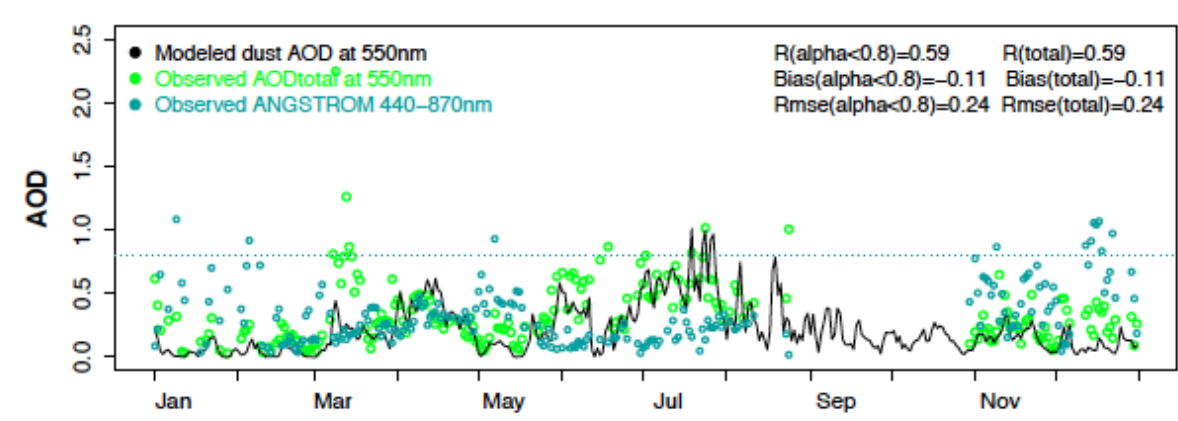

Nlamey
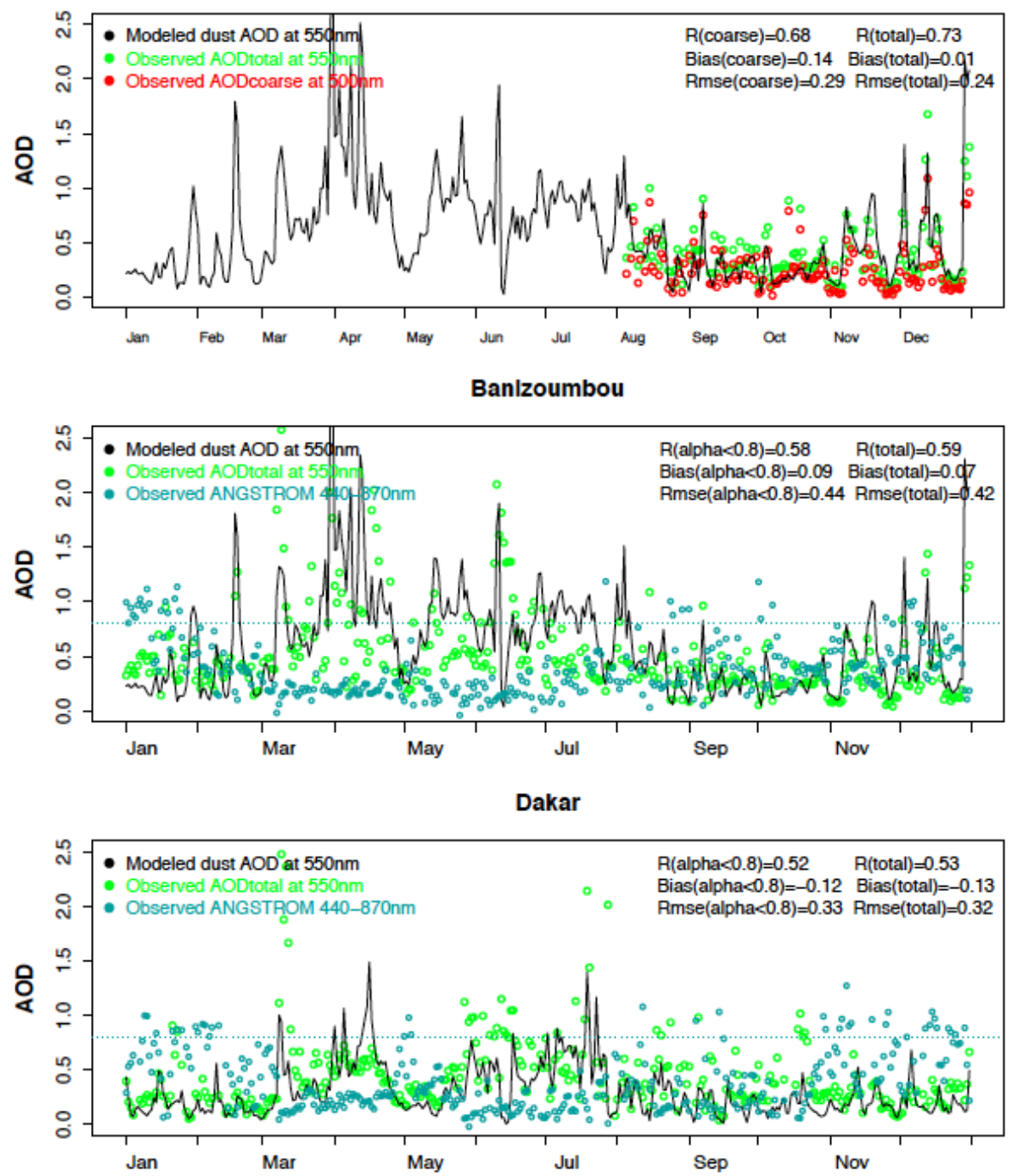


\section{Results: Gas-phase chemistry}

- Model setup:

$\rightarrow$ Global domain

$\rightarrow$ Non-hydrostatic physics

$\rightarrow 1.4^{\circ} \times 1^{\circ}$ horizontal resolution

$\rightarrow 64$ vertical (sigma-hybrid) layers

$\rightarrow 1^{\circ} \times 1^{\circ} \mathrm{NCEP} / \mathrm{FNL}$ analysis for meteorological initial conditions

$\rightarrow$ Chemistry initial conditions from LMDz-INCA

$\rightarrow$ Anthropogenic emissions: MOZART 2004

$\rightarrow$ Biogenic emissions: MEGAN online model

$\rightarrow$ No biomass burning emissions

$\rightarrow$ Half-year spin-up

$\rightarrow$ July - August 2004 simulation

$\rightarrow$ Regional domain

$\rightarrow$ Non-hydrostatic physics

$\rightarrow 12 \mathrm{~km} \times 12 \mathrm{~km}$

$\rightarrow 24$ vertical layers

$\rightarrow$ Anthropogenic emissions: EMEP 2004

$\rightarrow$ Biogenic emissions: MEGAN online model

$\rightarrow$ BC from global run

$\rightarrow$ August 2004 simulation

$\rightarrow$ All results are preliminary! 


\section{Preliminary evaluation with surface and ozonesondes}

- Evaluation against background sfc. from WDCGG, EMEP and CASTNET networks

- Evaluation from 3-hourly simulations
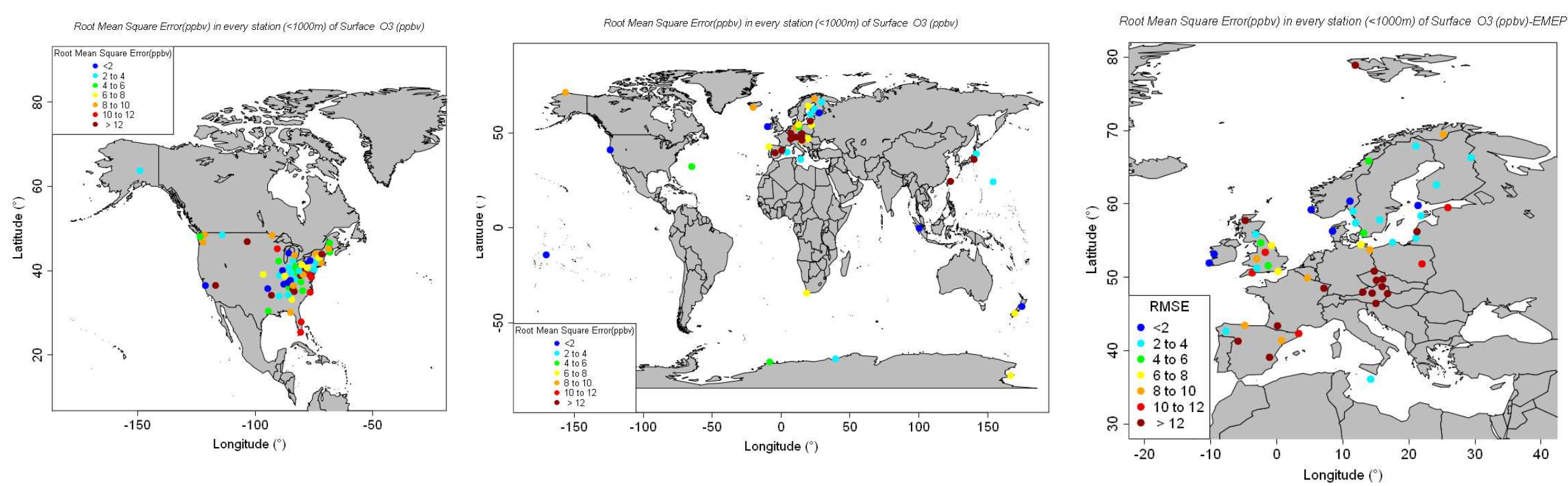

\begin{tabular}{|c|c|c|c|c|c|c|c|c|c|c|c|}
\hline GAS & $\begin{array}{c}\text { Mean mod } \\
\text { (ppbv) }\end{array}$ & $\begin{array}{c}\text { Mean obs } \\
\text { (ppbv) }\end{array}$ & NDATA & cor & Std (mod) & Std (obs) & RMSE (ppbv) & MB (ppbv) & MAE (ppbv) & MNBE (\%) & MNGE (\%) \\
\hline O3 WDCGG & 35.149 & 31.236 & 1243 & 0.27 & 12.7 & 8.8 & 13.4 & -1 & 9.9 & -0.2 & 24 \\
\hline O3 EMEP & 35.96 & 34.354 & 1882 & 0.27 & 13.8 & 8.4 & 14.1 & -1.7 & 11 & -2.5 & 26.9 \\
\hline O3 CASTNET & 38.755 & 31.959 & 1848 & 0.19 & 11.9 & 9.4 & 13.8 & 1.9 & 10.7 & 7.7 & 26 \\
\hline NO2 EMEP & 5.692 & 4.236 & 563 & 0.44 & 7 & 4.9 & 6.7 & 7.6 & 4.2 & 42.5 & 75.3 \\
\hline CO WDCGG & 177.194 & 122.963 & 791 & 0.32 & 93.3 & 54 & 107.6 & 56.4 & 67.8 & 75.9 & 81.5 \\
\hline
\end{tabular}




\section{RMSE (monthly data) OZONOSONDES (WOULLD, CMD and SHADOZ)}

\section{TROPO}

Root Mean Square Error(ppbv) for every station in the troposphere layer(10000m $\Rightarrow)($ (ppbv)

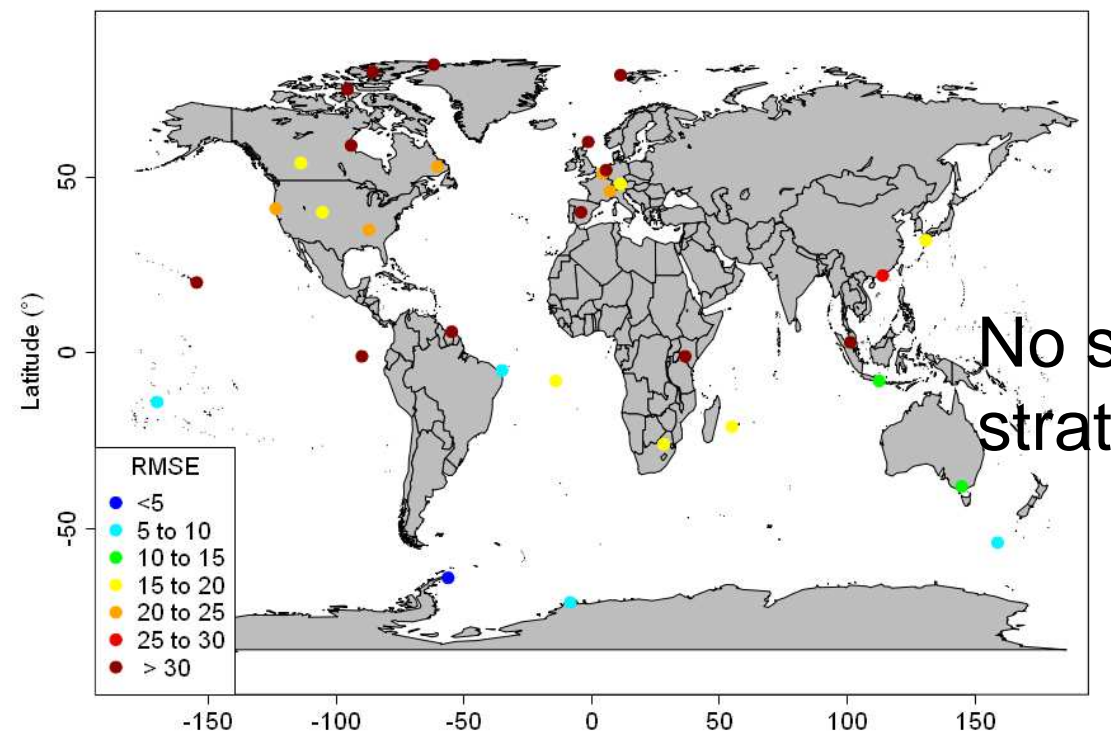

Root Mean Square Error(ppbv) for every station in the troposphere layer( $(10000 \mathrm{~m}=)(p p b v)$

\section{lower STRATO}

Root Mean Square Error(ppbv) for every station in the stratosphere layer $(10000 m \Rightarrow)(p p b v)$

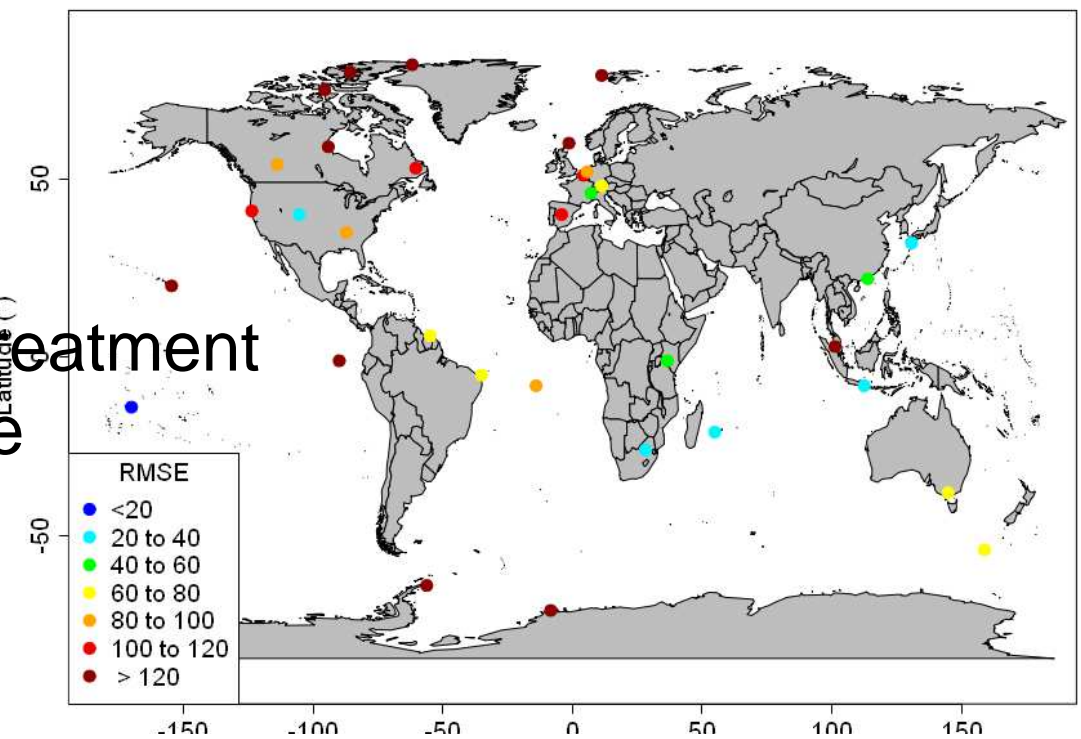

Root Mean Square Error(ppbv) for every station in the stratosphere layer(10000m $\Rightarrow)($ (ppbv)

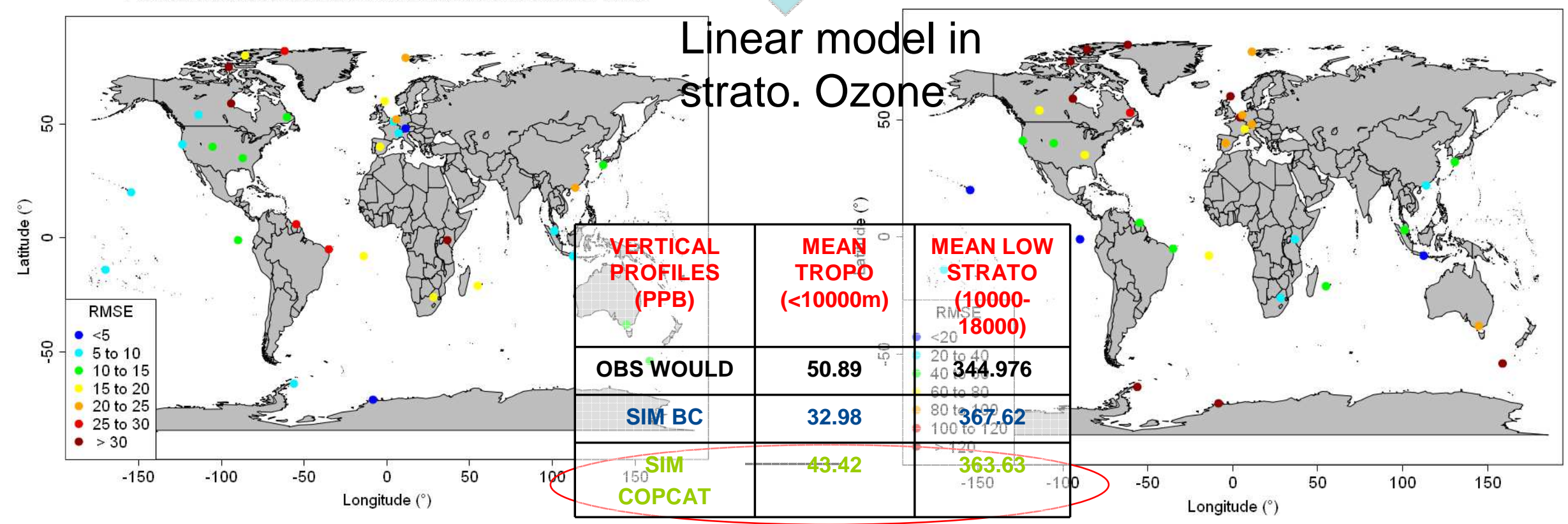




\section{Coupled global $\left(1^{\circ}\right)$ to regional $(12 \mathrm{~km})$ run}

Results for August months for global and regional runs - EMEP background stations

\begin{tabular}{|l|c|c|c|c|c|c|c|}
\hline \multicolumn{1}{|c|}{ Run } & Corr. & $\begin{array}{c}\text { MB } \\
\left(\mu \mathrm{g} / \mathrm{m}^{3}\right)\end{array}$ & $\begin{array}{c}\text { RMSE } \\
\left(\mu \mathrm{g} / \mathrm{m}^{3}\right)\end{array}$ & $\begin{array}{c}\text { MNBE } \\
(\%)\end{array}$ & $\begin{array}{c}\text { MNGE } \\
(\%)\end{array}$ & $\begin{array}{c}\text { MFB } \\
(\%)\end{array}$ & $\begin{array}{c}\text { MFE } \\
(\%)\end{array}$ \\
\hline Global & 0.33 & -12.9 & 41.4 & -11.5 & 30.9 & -21.3 & 36.7 \\
\hline Regional & 0.51 & 0.7 & 23.1 & 2.1 & 17.2 & -0.3 & 17.1 \\
\hline
\end{tabular}
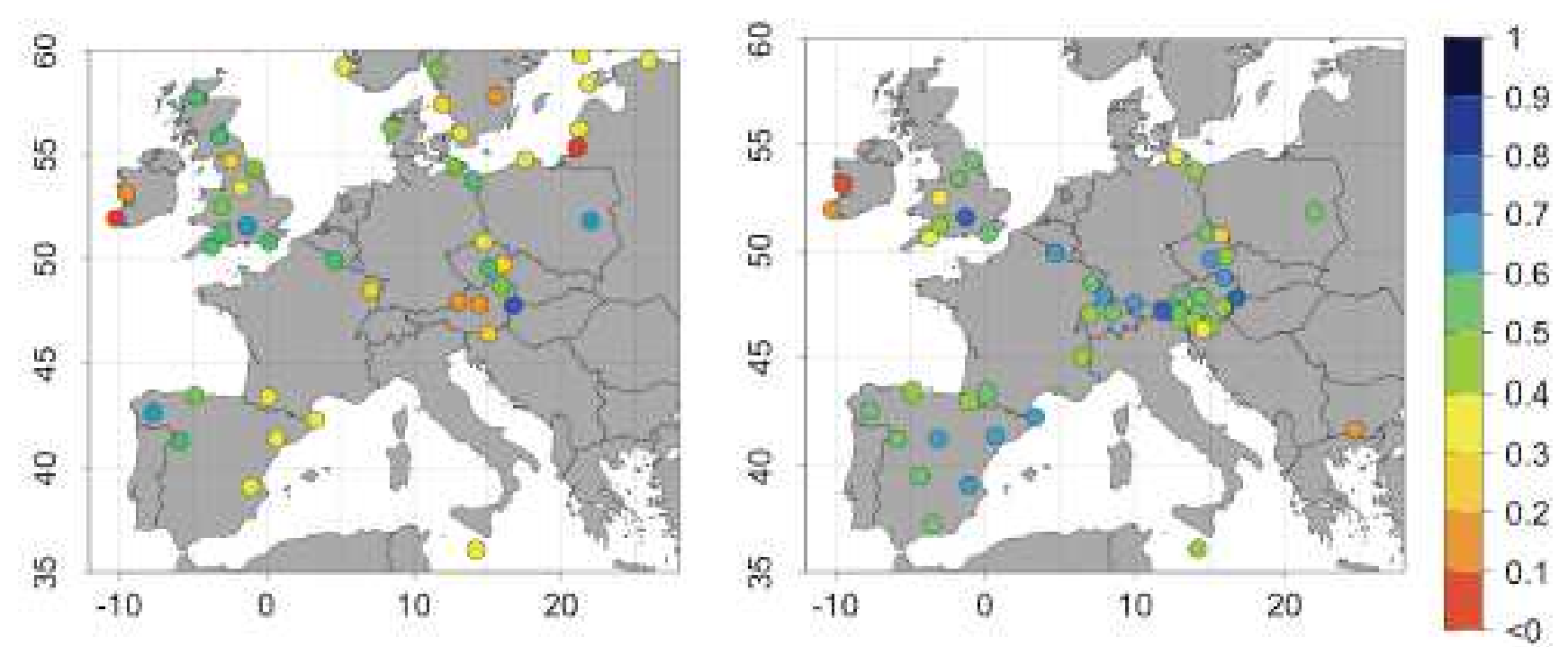

Surface ozone correlation at several EMEP stations - Global (left) and Regional (right) 


\section{Regional run: Model vs Obs}

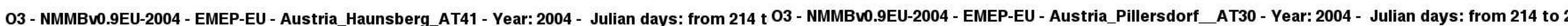
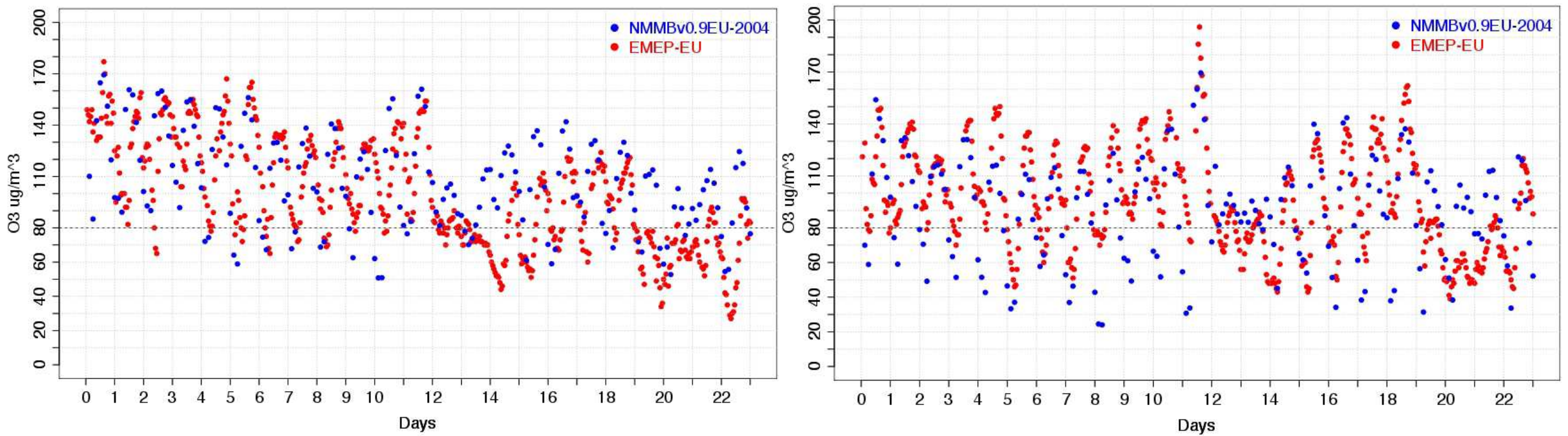

- NMMBv0.9EU-2004 - EMEP-EU - Netherlands_Kollumerwaar_NL09 - Year: 2004 - Julian days: from 21 SO2 - NMMBv0.9EU-2004 - EMEP-EU - Spain_O_Savinao_ES16 - Year: 2004 - Julian days: from 214 to 2
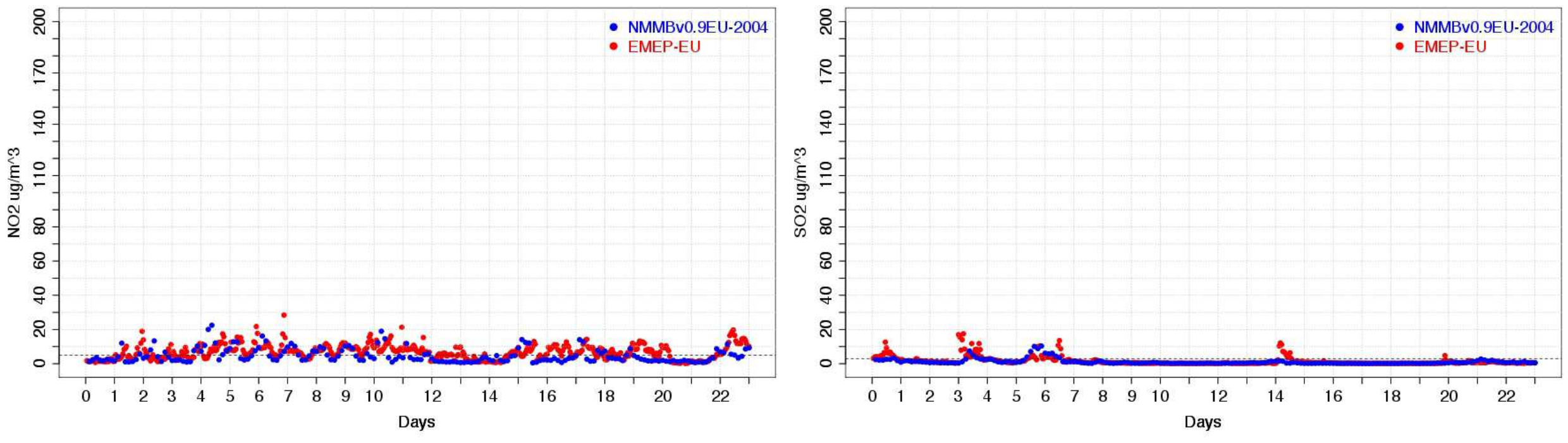

- NMMBv0.9EU-2004 


\section{Sea Salt results (Spada et al., 2012) - Global run 2006 at $1^{\circ} \times 1.4^{\circ}-$ AOD}
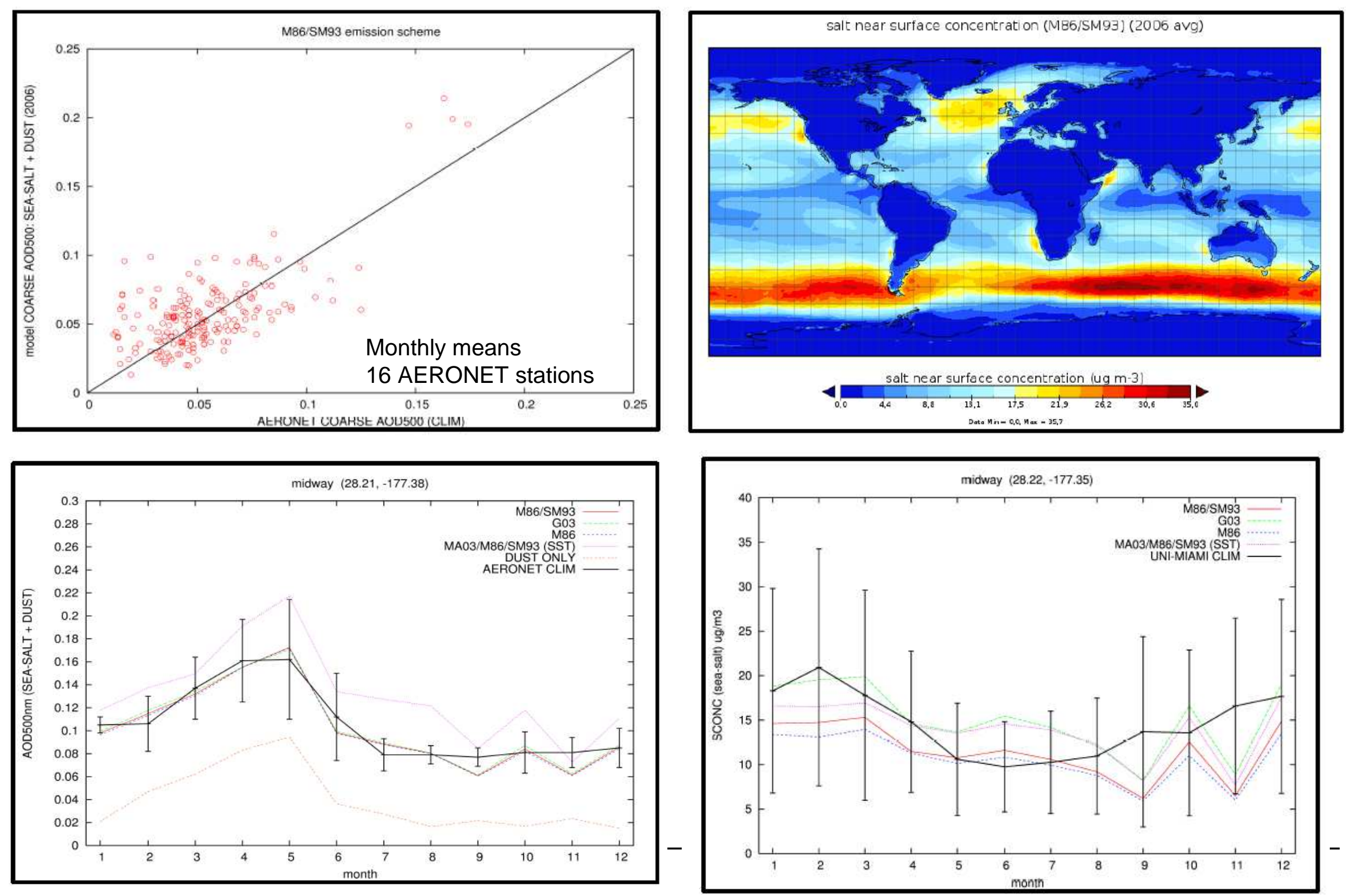


\section{NMMB/BSC-CTM sea-salt: multiscale simulation}

- Same emission scheme

- Consistent physics from global to regional

- Fully on-line aerosol coupling

- Modeled sea-salt concentrations is coastal areas may be strongly affected by resolution

- Global - to - regional: increased capability to resolve both SSA deposition scales and meso circulations

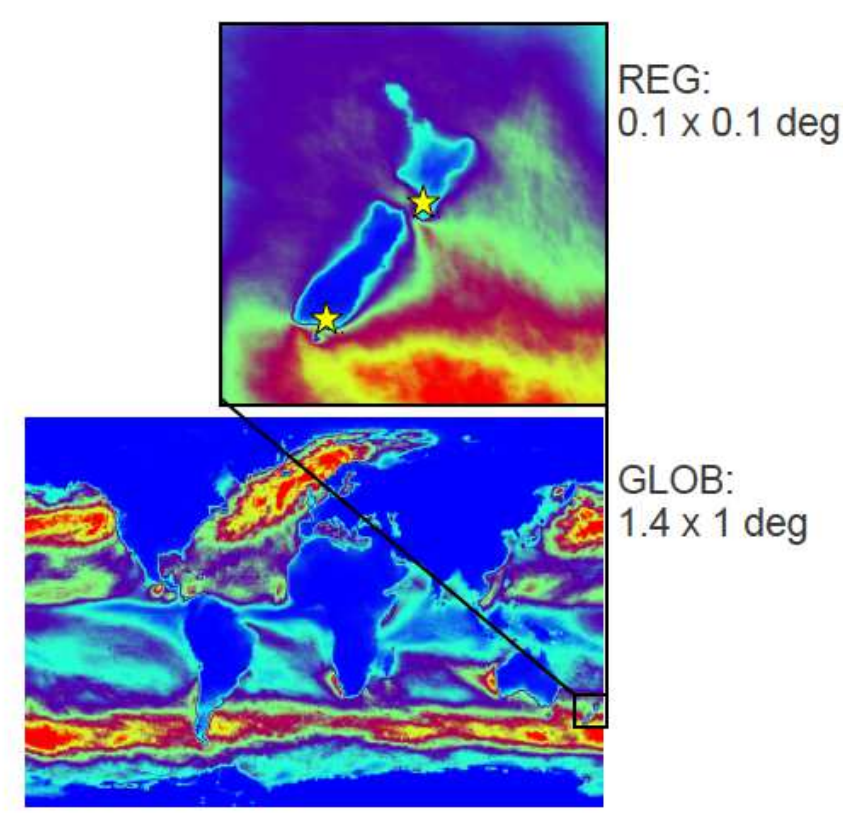

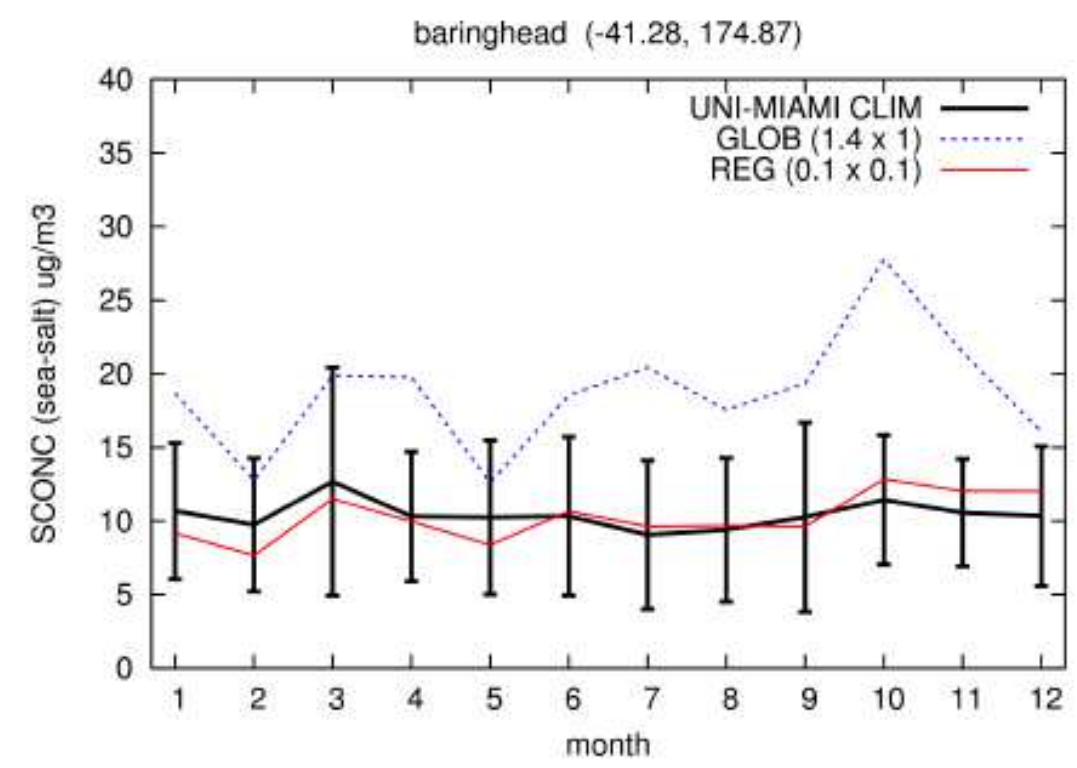

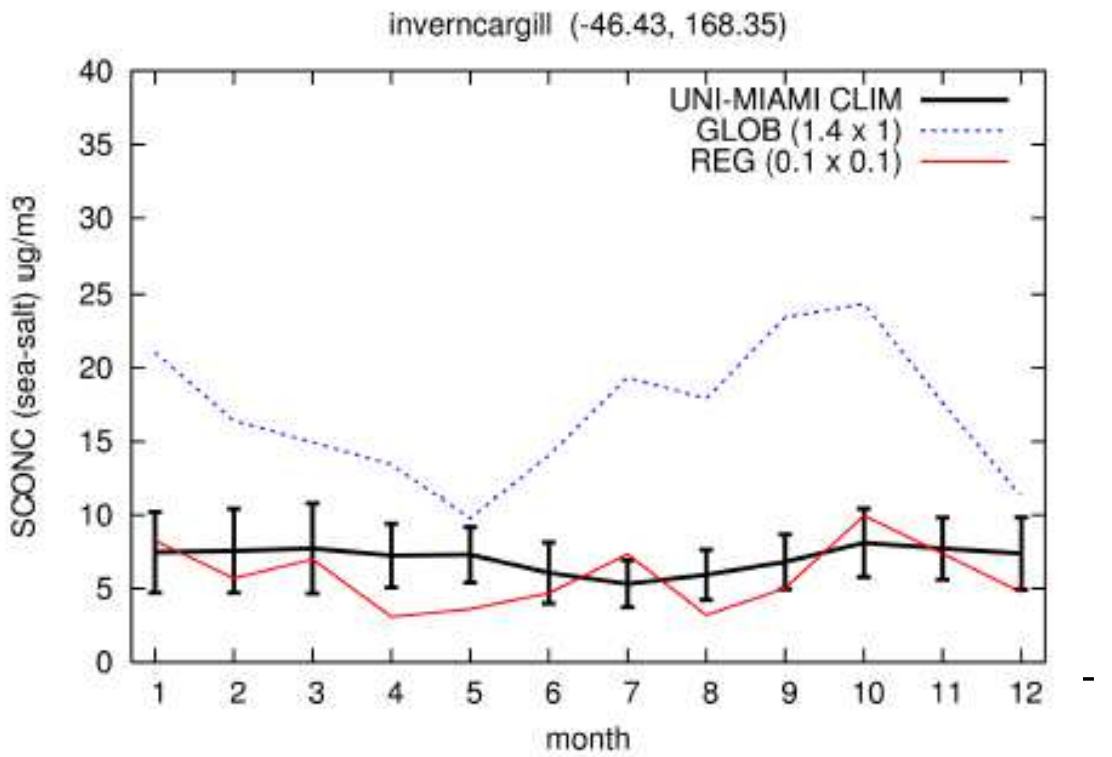


- Future developments 


\section{Future developments (I/II)}

- Improvement and evaluation of the chemistry part of the model.

- Implementation of the other global relevant aerosol species, i.e. black (BC) and organic carbon (OC), and sulfate (SO4), in addition to dust (DU) and sea salt (SS).

- It is planned to couple the radiative scheme with all the considered aerosol species to simulate the direct aerosol radiative effect.

- It is planned to couple the model ozone prediction with the radiative scheme of NMMB.

- It is panned to couple the photolysis scheme with the model clouds, ozone, and aerosol species (DU, SS, BC, OC, SO4). 


\section{Future developments (II/II)}

- Implementation of secondary aerosol schemes (SIA, new SOA parameterizations) for LAM applications at high-resolutions

- Evaluation of the gas-phase chemistry on regional domains

- Experimental dust forecasts on global and regional domains within the SDS-WAS system 


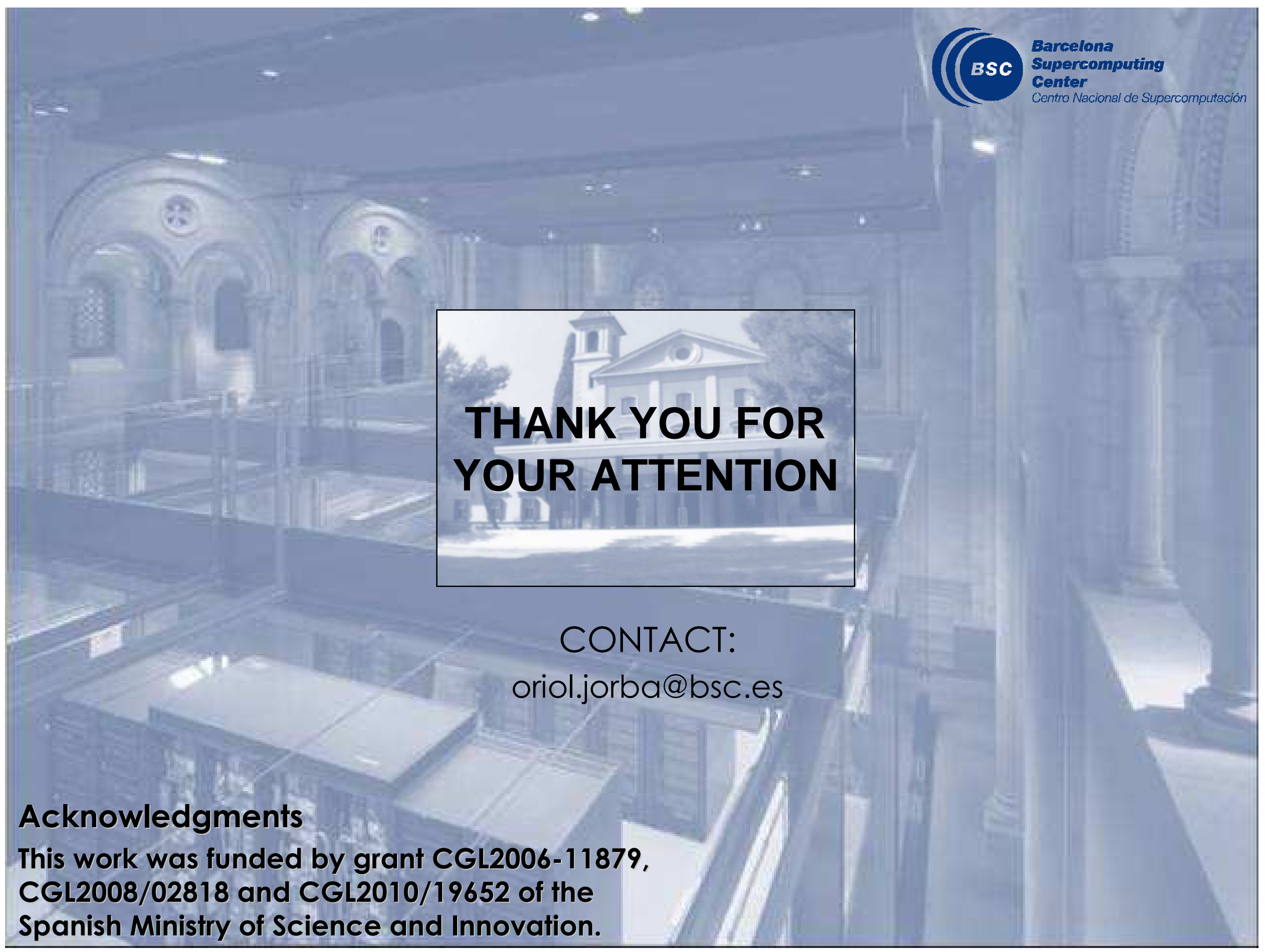

